Acoustic properties of porous microlattices from effective medium to scattering dominated regimes

Sebastian Krödel, Antonio Palermo, and Chiara Daraio

Citation: The Journal of the Acoustical Society of America 144, 319 (2018); doi: 10.1121/1.5046068

View online: https://doi.org/10.1121/1.5046068

View Table of Contents: http://asa.scitation.org/toc/jas/144/1

Published by the Acoustical Society of America 


\title{
Acoustic properties of porous microlattices from effective medium to scattering dominated regimes
}

\author{
Sebastian Krödel \\ Department of Mechanical and Process Engineering, ETH Zürich, Tannenstrasse 3, 8092, Zürich, Switzerland \\ Antonio Palermo a) \\ Department of Civil, Chemical, Environmental and Materials Engineering, University of Bologna, \\ Viale del Risorgimento 2, Bologna 40136, Italy \\ Chiara Daraio ${ }^{\text {b) }}$ \\ Division of Engineering and Applied Science, California Institute of Technology, \\ 1200 California Boulevard, Pasadena, California 91125, USA
}

(Received 13 October 2017; revised 13 June 2018; accepted 24 June 2018; published online 24 July 2018)

\begin{abstract}
Microlattices are architected materials that allow for an unprecedented control of mechanical properties (e.g., stiffness, density, and Poisson's coefficient). In contrast to their quasi-static mechanical properties, the acoustic properties of microlattices remain largely unexplored. This paper analyzes the acoustic response of periodic millimeter-sized microlattices immersed in water using experiments and numerical simulations. Microlattices are fabricated using high-precision stereolithographic three-dimensional printing in a large variety of porosities and lattice topologies. This paper shows that the acoustic propagation undergoes a frequency dependent transition from a classic poroelastic behaviour that can be described by Biot's theory to a regime that is dominated by scattering effects. Biot's acoustic parameters are derived from direct simulations of the microstructure using coupled fluid and solid finite elements. The wave speeds predicted with Biot's theory agree well with the experimental measures. Within the scattering regime, the signals show a strong attenuation and dispersion, which is characterized by a cut-off frequency. The strong dispersion results in a frequency dependent group velocity. A simplified model of an elastic cylindrical scatterer allows predicting the signal attenuation and dispersion observed experimentally. The results in this paper pave the way for the creation of microlattice materials for the control of ultrasonic waves across a wide range of frequencies. (C) 2018 Acoustical Society of America.
\end{abstract}

https://doi.org/10.1121/1.5046068

$[\mathrm{KVH}]$

Pages: 319-329

\section{INTRODUCTION}

The behaviour of acoustic waves propagating in heterogeneous materials depends on the ratio between their wavelength and the length scale of the inhomogeneity or scatterer. For instance, $\mathrm{kHz}$ sound waves in a solid can be described by elastic continuum theory, while the description of $\mathrm{THz}$ thermal phonons in the same material requires the knowledge of the exact molecular structure of the crystal. ${ }^{1,2}$ The transition between the different regimes of wave propagation is of great technological importance: thermal transport can undergo a transition from wave-like to diffusive transport that we perceive on macroscopic scales. Such transitions from quasihomogeneous to scattering dominated behaviour also occur in acoustical systems. ${ }^{3}$ In microstructured acoustic systems strong scattering can lead to dramatically altered acoustic transmission and frequency dependent group velocity. ${ }^{4}$

An emerging class of microstructured materials are microlattices that can be designed to obtain material

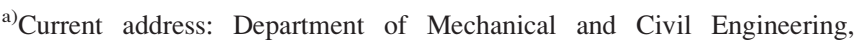
California Institute of Technology, 1200 California Boulevard, Pasadena, CA 91125 , USA.

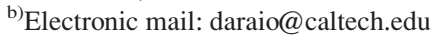

properties which are unobtainable in natural solid materials such as negative Poisson's ratio ${ }^{5}$ or zero-shear module ${ }^{6,7}$ and dilatation metamaterials. ${ }^{8}$ As microlattice materials are composed of a periodic arrangement of slender truss elements, they can be interpreted as perfectly ordered open-cell foams. The acoustics of fluid saturated cellular foams at low frequencies are described by Biot's effective medium theory, ${ }^{9,10}$ which predicts the occurrence of two kinds of longitudinally polarized waves: a fast wave $c_{p 1}$ where the cellular frame and the fluid in the pores move in-phase, and a slow wave $c_{p 2}$ where they move out-of-phase. Biot's theory has been successfully applied to waves across scales ranging from cancellous bone ${ }^{11,12}$ to seismic waves. ${ }^{13}$ An important step towards its application is the calculation of all the required physical parameters directly from the complex porous microstructure. This process is mainly carried out for rigid microstructures ${ }^{14,15}$ in the field of audible acoustics to derive effective medium theories of the compressible fluid in the pores. ${ }^{16}$ A fundamental limitation of Biot's theory is the continuum assumption, which is valid only for low frequency waves with wavelengths much larger than the pore size. For shorter wavelengths, scattering effects play a role. Strong scattering phenomena have been extensively studied in granular media., ${ }^{3,17}$ Materials composed of random 
arrangements of spherical solid particles dispersed in a fluid matrix have been successfully used to realize acoustic analogies of Anderson localization, ${ }^{18}$ electron tunneling, ${ }^{19}$ and negative index of refraction. ${ }^{20}$ Scattering effects can be subwavelength in the fluid matrix ${ }^{21}$ if the wavelength is drastically shortened within the scatterer or if the scatterer undergoes low frequency structural modes, such as Minaert bubble resonances. $^{22}$ In contrast, for open cellular materials, scattering effects have only been considered in the limit of weak scattering ${ }^{23,24}$ or low frequencies. ${ }^{25}$ Therefore, the impact of strong scattering on wave propagation in cellular materials and the physical mechanisms beyond the continuum description of Biot are less well understood. ${ }^{26}$ Microlattices can serve as a model material with controlled microarchitecture and used to study scattering effects in a highly controllable system. Moreover, as cellular architectures frequently occur in natural and artificial materials, the correct interpretation of their acoustic footprint is important for applications in biomedical imaging and nondestructive testing.

Here we show that ultrasonic wave propagation in microlattice materials can be described by either continuum or scattering theory depending on the carrier frequency and the truss radius. The splitting of wave propagation into different propagation regimes as a function of grain radius has been observed for non-linear granular media; ${ }^{27,28}$ however, it has not been shown for foam-like structures. We fabricate millimetre-sized lattices using high precision stereolithographic (SLA) three-dimensional (3D) printing and experimentally measure $c_{p 1}$-waves at low frequencies in microlattice samples with varying porosity and topology. We employ coupled solid-fluid Bloch-Finite Element (FE) simulations with periodic boundary conditions of the form $(\mathbf{u}, p)^{+}=\exp (i \mathbf{k} \cdot \mathbf{x})(\mathbf{u}, p)^{-}$, where $(\mathbf{u}, p)^{+,-}$are the solid displacement and fluid pressure field on the boundaries of the unit cell and $\mathbf{k}$ denotes the wave vector. Using these simulations, we predict the microlattices' fast wave speeds, which are in excellent agreement with the experimental measurements. At high frequency, the continuum description breaks down and scattering effects lead to a distinct cut-off frequency with strong signal attenuation. The experimental measured group velocities past the cut-off are decreased by up to $40 \%$ when compared to Biot's theory. The extremely slow waves are the result of the interaction of scatterers in the medium. ${ }^{29}$ The cut-off frequency is independent of lattice topology and robust against random perturbations of the periodicity. We show that the cross-over frequency can be well predicted by a simple multiple scattering model and Bloch-FE simulations that incorporate the effect of the elastic frame.

\section{MICROLATTICE FABRICATION}

We fabricate Kelvin and Diamond-like microlattices with bending dominated topology [Figs. 1(a) and 1(b)] and Octet lattices with stretch dominated topology [Fig. 1(c)] using micro-SLA fabrications techniques (Autodesk Ember ${ }^{\mathrm{TM}}$ ). The connectivity of the lattices, and therefore the dominant deformation mechanism, has an important impact on the macroscopic mechanical properties such as stiffness or strength; ${ }^{30}$ however, the impact on the acoustical properties is not clearly understood. We use the PR $48^{\mathrm{TM}}$ base material, which is a commercial acrylic photoresist, and measure a compressional wave speed of $c_{p}=2380 \mathrm{~m} / \mathrm{s}$, a shear wave speed of $c_{s}=1000 \mathrm{~m} / \mathrm{s}$, and a density of $\rho_{s}=1190 \mathrm{~kg} \mathrm{~m}^{-3}$.

To vary the porosity, we vary the aspect ratio between the truss radius $r$ and the truss length $L$. Trusses are either $1 \mathrm{~mm}$ or $0.66 \mathrm{~mm}$ in length and have a radius between $0.15 \mathrm{~mm}$ and $0.4 \mathrm{~mm}$. The microlattice samples show a large range of porosities ranging from $54 \%$ to $98 \%$ [Fig. 1(d)]. Stretch dominated lattices have a significantly lower porosity at the same aspect ratio due to the larger connectivity of the lattice. We fabricate lattice samples with an overall dimension of $35 \times 25 \times 25 \mathrm{~mm}^{3}$ consisting of a large number of unit cells ( $\approx 2000,18$ unit cell in the $z$-direction).

\section{LOW FREQUENCY PROPAGATION}

\section{A. Experimental measurements of fast Biot waves}

To study acoustic wave propagation in the fluid saturated lattices, we immerse our structures in water (with wave speed $c_{w}=1481 \mathrm{~m} / \mathrm{s}$ ), between a broadband ultrasonic transducer (V391-SU centered at $0.5 \mathrm{MHz}$ for low-frequency and V394$\mathrm{SU}$ centered at $1 \mathrm{MHz}$ for high frequency measurements) and a hydrophone (Precision Acoustics, diameter $=4 \mathrm{~mm}$ ), see Fig. 1(e). Signals are generated by the arbitrary waveform generator of a Lock-In amplifier (UHFLI, Zurich Instruments) connected to a radio-frequency amplifier (Amplifier Research 75A220). To study low-frequency Biot waves, we transmit short pulses (1 cycle) at $200 \mathrm{kHz}$ with a wavelength of $1.5 \mathrm{~cm}$ in the fluid, much larger than the characteristic pore size of the lattices. Typical measurements are shown in the inset in Fig. 1(e). We observe the arrival of a compact wave packet, which corresponds to the fast Biot wave. The arrival of the wave packet is shifted to earlier times for lower porosities, indicating an increasing wave speed. To estimate the Biot wave speed, we measure the time of flight for pulses travelling through each lattice [Figs. 2(a)-2(c)].

The experimental results show wave speeds ranging between $1500 \mathrm{~m} / \mathrm{s}$ and $1780 \mathrm{~m} / \mathrm{s}$ for $c_{p 1}$ waves, see Figs. 2(a)-2(c). Stretch dominated lattices [Figs. 2(a) and 2(b)] show a larger variability as their mechanical properties scale stronger with a varying porosity. We are not able to measure slow Biot waves due to their highly dissipative nature. ${ }^{12}$

\section{B. Experimental attenuation at low frequencies}

In addition to the wave speed of the propagating fast waves we measure the attenuation coefficient $\alpha_{l}$ defined as

$$
\alpha_{l}=\frac{20 \log _{10} \frac{S_{w}}{S_{l}}}{L}
$$

where $S_{w}$ is the transmitted amplitude through water and $S_{l}$ is the transmitted amplitude through the microlattice with length $L$. Experiments were carried out by propagating short cycles at four different frequencies between 100 and 400 $\mathrm{kHz}$. The experimental outcomes show a significant dependence of the attenuation on the porosity [see Figs. 2(d)-2(f)]. 
(a)

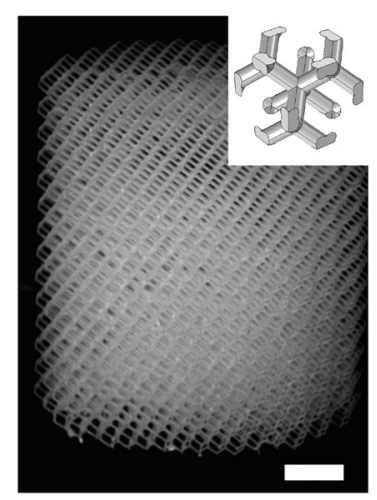

(d)

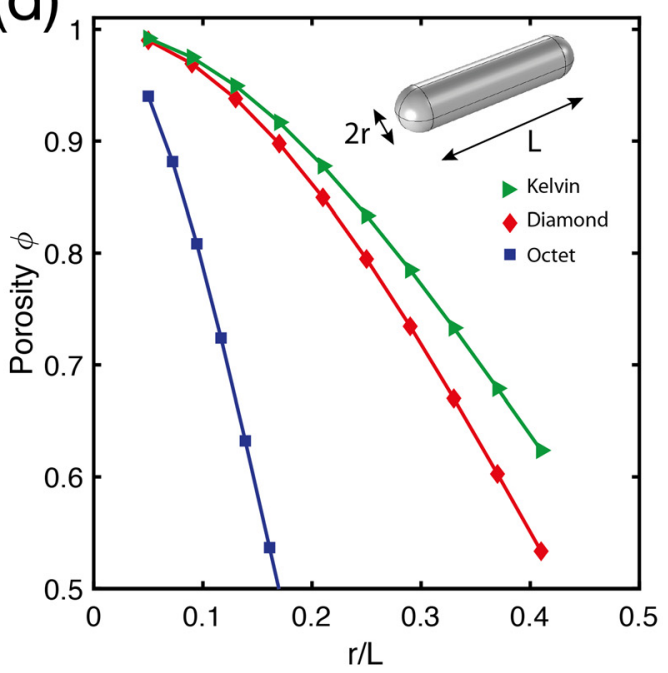

(b)

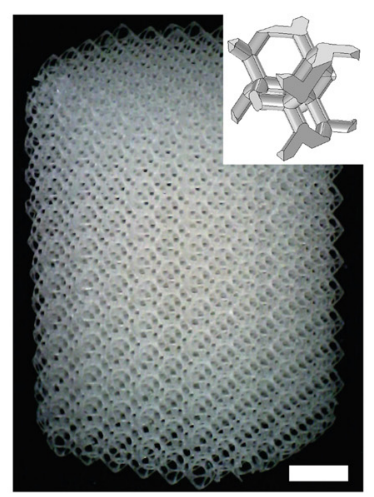

(c)

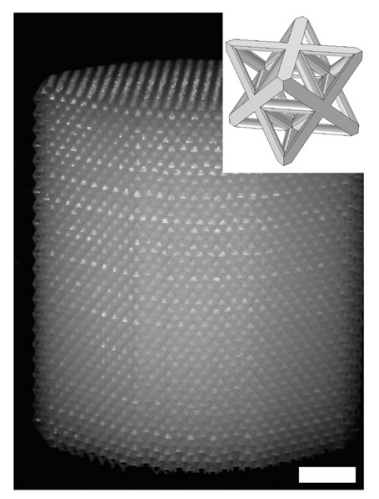

(e)

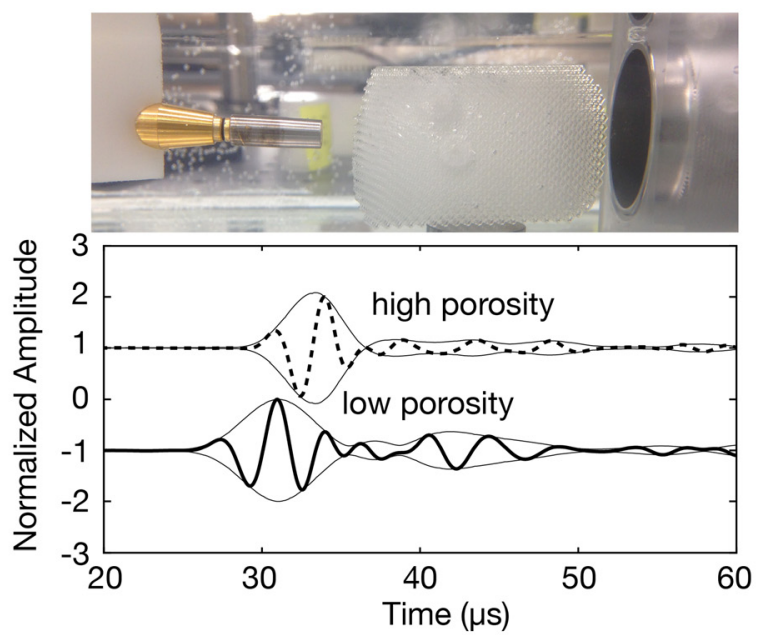

FIG. 1. (Color online) Microlattices fabricated using stereolithographic 3D printing. (a) Photograph of fabricated Diamond lattice, (b) Kelvin lattice, and (c) Octet lattice. The scale bar is $2 \mathrm{~mm}$. (d) Porosity of the analysed lattices as a function of the truss aspect ratio $r / L$. (e) Experimental setup including a Hydrophone (left) and a broadband ultrasonic transducer (right). The bottom panel shows typical waveforms obtained for low frequency (100 kHz) measurements for high and low porosity samples. Signals have been normalised by their maximum amplitude to ease visualisation.

A lower porosity results in significantly larger signal attenuation up to $1000 \mathrm{~dB} / \mathrm{m}$ for the lowest porosity Octet lattice [Fig. 2(f)]. The dependence on the frequency in the long wavelength regime is low. Especially for the Diamond and Kelvin structures a slightly increasing attenuation between 100 and $400 \mathrm{kHz}$ is detectable [see Figs. 2(d) and 2(e)].

\section{Numerical predictions of fast Biot wave speeds and attenuation}

We compare the experimental measurements for fast waves to the predictions of Biot's theory. The governing equations for Biot's theory are $^{31}$

$$
\begin{aligned}
& -\hat{\rho}_{11} \omega^{2} \hat{\boldsymbol{u}}-\hat{\rho}_{12} \omega^{2} \hat{\boldsymbol{U}} \\
& \quad=(P-G) \nabla(\nabla \cdot \hat{\boldsymbol{u}})+Q \nabla(\nabla \cdot \hat{\boldsymbol{U}})+G \nabla^{2} \hat{\boldsymbol{u}}, \\
& -\hat{\rho}_{22} \omega^{2} \hat{\boldsymbol{U}}-\hat{\rho}_{12} \omega^{2} \hat{\boldsymbol{u}}=R \nabla(\nabla \cdot \hat{\boldsymbol{U}})+Q \nabla(\nabla \cdot \hat{\boldsymbol{u}}),
\end{aligned}
$$

where $\hat{\boldsymbol{u}}$ and $\hat{\boldsymbol{U}}$ denote the volume average solid and fluid displacement fields; $P, G, Q$, and $R$ are generalized elastic constants; and $\hat{\rho}_{11}, \hat{\rho}_{22}$, and $\hat{\rho}_{12}$ are frequency dependent solid, fluid, and coupling density terms. The constants $(P, G$, $Q, R)$ appearing in the equations of motion are derived from measurable quantities such as the porosity $\phi$, the bulk modulus of the fluid, the bulk modulus of the solid and fluid material $K_{s}, K_{f}$, the shear modulus of the solid $G$, and the effective compressional modulus of the microlattice frame $K_{l}$. The parameters that are required to solve Eqs. (2) and (3) are derived by modelling the microstructure directly in a finite element framework using Comsol Multiphysics. The first parameter we calculate is the bulk compressional module of the drained lattice $K_{l}$. We simulate the drained lattices with varying porosities [see inset Fig. 3(a)] assuming 3D linear elasticity. To obtain the characteristic dispersion relation for each lattice type, we apply periodic Floquet boundary conditions on the sites of the cubic unit cell. Subsequently, we vary the wave vector $k_{z}$ and solve for the eigenfrequencies. The dispersion describes the propagation of acoustic waves in the solid frame in the $z$-direction. In the nondispersive long-wavelength regime $\left(\lambda=2 \pi / k_{z} \gg a\right.$, where $a$ denotes the size of the unit cell), the shear and pressure wave speeds are related to the gradient of the frequency with respect to the wave number 


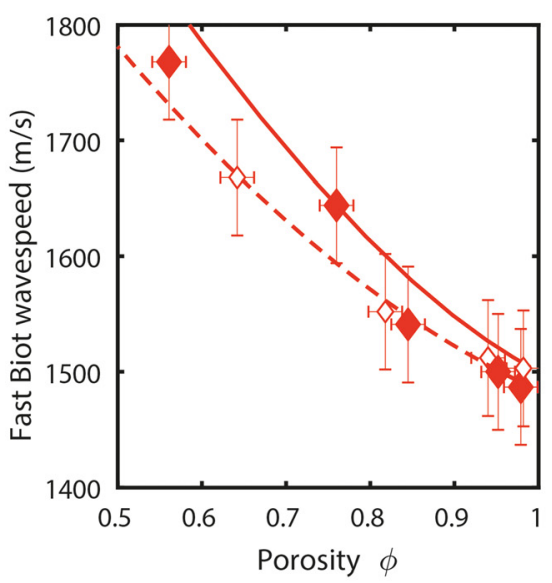

(d)

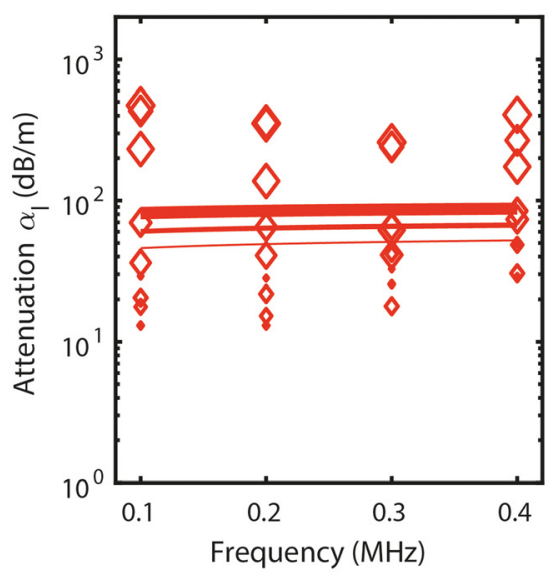

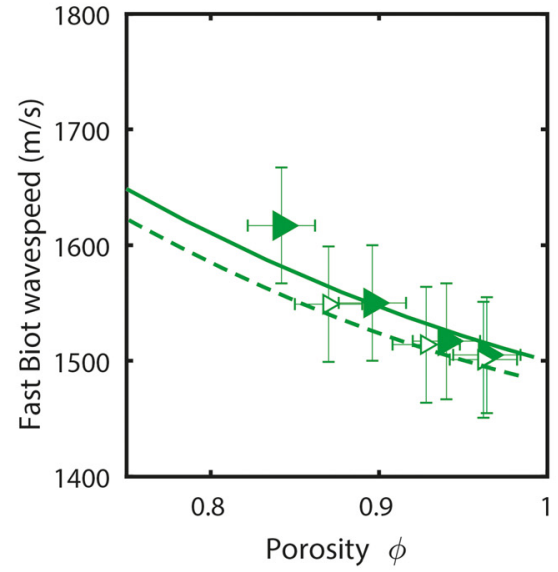

(e)

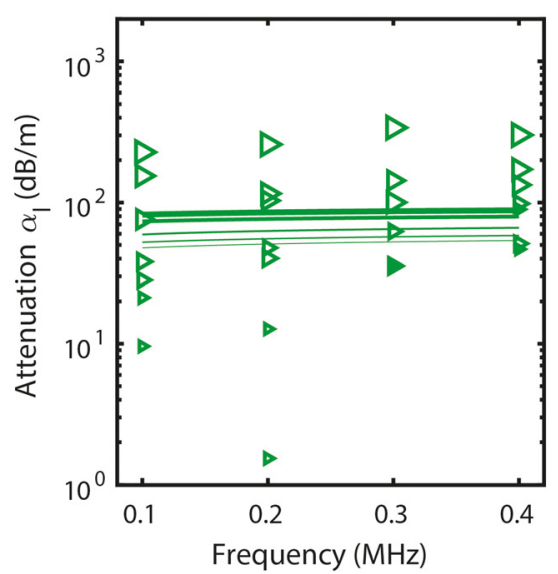

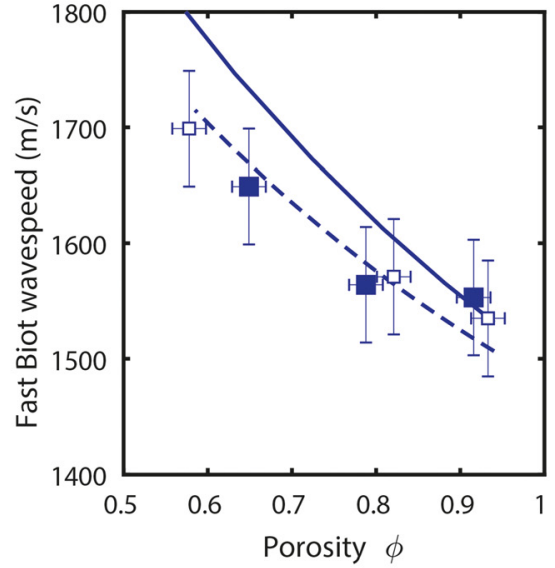

(f)

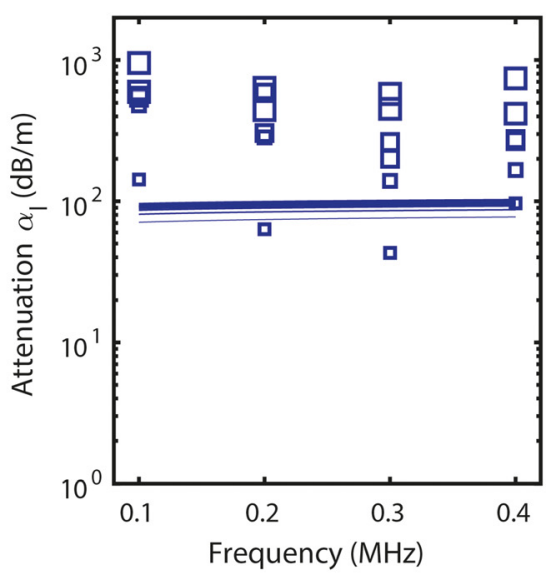

FIG. 2. (Color online) Fast Biot wave speeds as a function of porosity and experimental attenuation at low frequencies for (a) Diamond lattices, (b) Kelvin lattices, and (c) Octet lattices. Markers represent experiments at $200 \mathrm{kHz}$, solid lines analytic Biot's theory, and dashed lines finite element simulations. Hollow markers are lattices with $L=0.66 \mathrm{~mm}$, solid markers are lattices with $L=1 \mathrm{~mm}$. The error bars represent the error in the calculated porosities due to variations in the fabrication process and the uncertainty in determining the peak amplitude in time. Experimentally measured and analytically calculated attenuation coefficients for (d) Diamond lattices, (e) Kelvin lattices, and (f) Octet lattices. Markers are used for experimental data. The size of the marker is proportional to the porosity, where a small size represents high porosity and a large size low porosity with an overall range between $1.8 \%$ and $42.2 \%$. The lines of the analytical calculations are also scaled according to the same porosities.

$$
\left.\frac{\partial \omega_{p, s}}{\partial k_{z}}\right|_{k_{z}=0}=c_{p, s}
$$

where $\omega_{p, s}$ is the angular frequency of the pressure, shear waves in the drained lattice. By assuming linear-elastic and isotropic behaviour, the wave speeds are related to the drained elastic constants and the solid density $\rho_{s}$ : the Young's modulus of the lattice, $E_{l}$, shear module of the lattice, $G_{l}$, compressibility of the lattice, $K_{l}$ and Poisson's ratio of the lattice, $\nu_{l}$,

$$
\begin{aligned}
& \nu_{l}=\frac{1-2\left(c_{s} / c_{p}\right)^{2}}{2-2\left(c_{s} / c_{p}\right)^{2}}, \\
& E_{l}=\frac{c_{p}^{2}(1-\phi) \rho_{s}\left(1+\nu_{l}\right) /\left(1-2 \nu_{l}\right)}{1-\nu_{l}}, \\
& G_{l}=c_{s}^{2}(1-\phi) \rho_{s}
\end{aligned}
$$

$$
K_{l}=\frac{E_{l}}{3\left(1-2 \nu_{l}\right)} .
$$

To calculate the permeability, $\kappa_{0}$, under quasi-static conditions, we assume a laminar flow throughout the lattice for an applied pressure drop, $\Delta P$, along the $z$-direction [see inset Fig. 3(b)]. To account for continuity, we apply periodic boundary conditions in the $x$ - and $y$-directions. According to Darcy's law, the flow in the $z$-direction through porous media under static conditions is governed by

$$
\phi V_{z}=-\frac{\kappa_{0}}{\mu} \nabla p,
$$

where $V_{z}$ is the average flow velocity in the $z$-direction, $\mu$ is the dynamic viscosity of water $\left(\mu_{w}=1 \times 10^{-3}\right)$, and $\nabla p$ is the pressure gradient. The average flow velocity can be calculated by integration over the fluid area in the $z$-direction with unit vector $\mathbf{e}_{\mathbf{z}}$, 
(a)
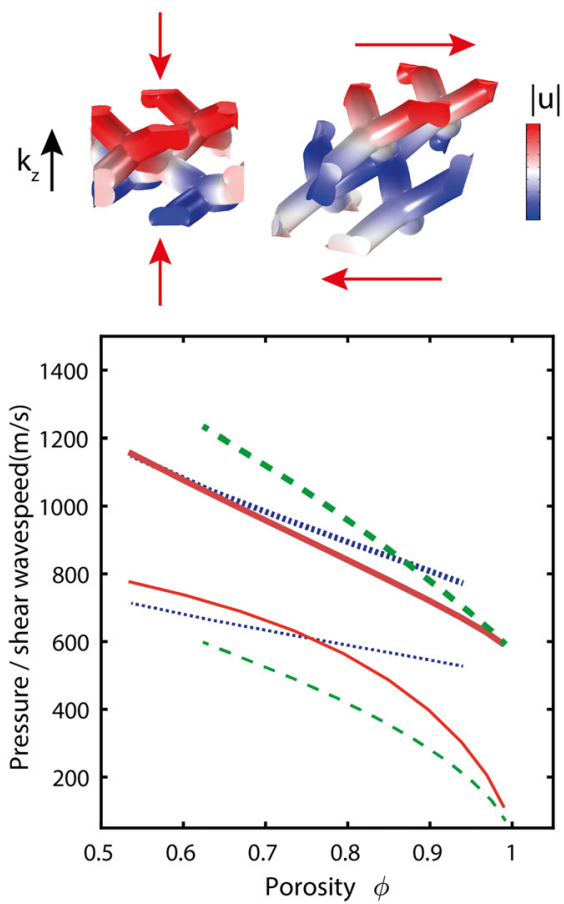

(b)

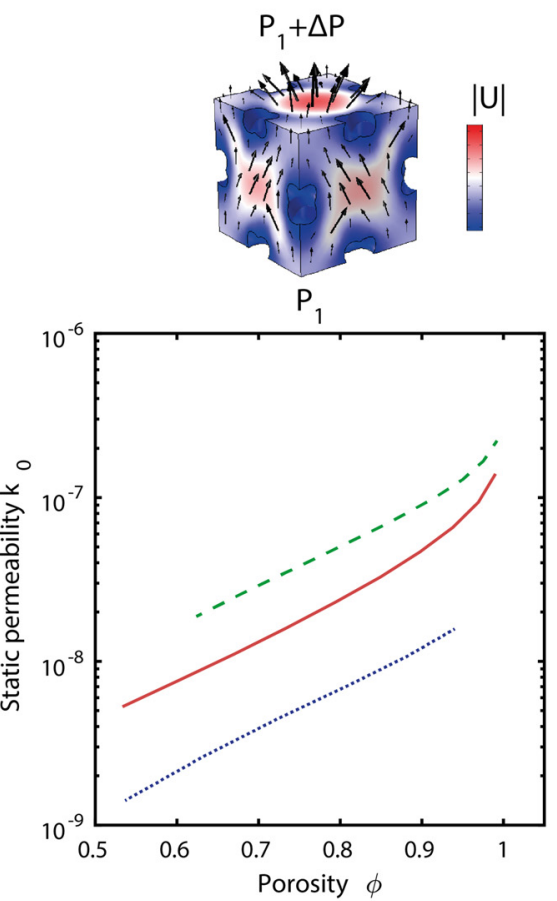

(c)
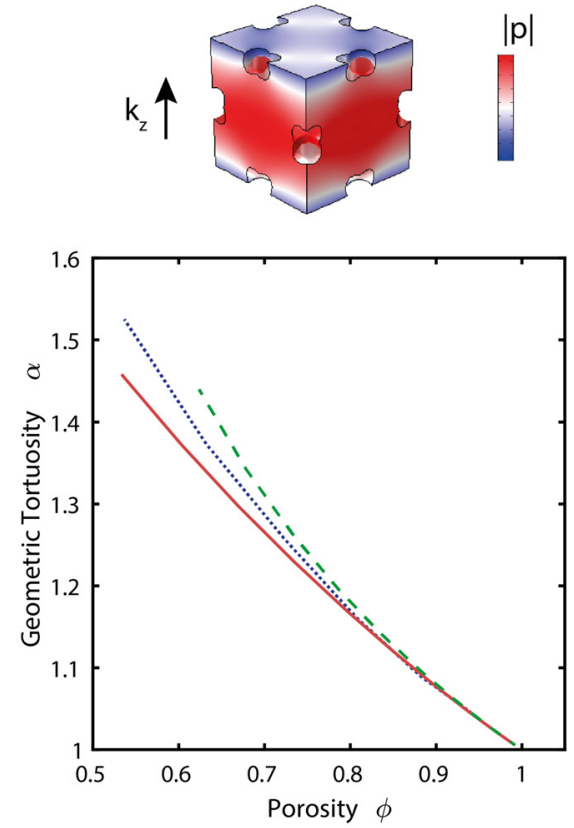

FIG. 3. (Color online) Calculation of parameters for Biot wave speeds. (a) Pressure (thick lines) and shear wave speeds (thin lines) of the drained lattices: Diamond (solid, red), Kelvin (dashed, green), Octet (pointed, blue) as a function of porosity. Inset shows modal displacement fields for the longitudinal and shear wave, the color intensity ranges from minimal to maximal absolute displacement (b) Static permeability of the lattices: Diamond (solid, red), Kelvin (dashed, green), Octet (pointed, blue) as a function of porosity. Inset shows the absolute flow velocity in the cubic unit cell for the applied pressure drop. (c) Geometric tortuosity of the lattices: Diamond (solid, red), Kelvin (dashed, green), Octet (pointed, blue) as a function of porosity. Inset shows the pressure distribution for the longitudinal acoustic mode propagating in the $z$-direction for the rigid lattice case.

$$
V_{z}=\frac{1}{A_{z}} \int_{A_{z}} \boldsymbol{V} \cdot \mathbf{e}_{z} \mathrm{~d} A .
$$

For an applied pressure drop of $\Delta P$ across the finite size unit cell of length $a$, we can express the permeability

$$
\kappa_{0}=\frac{\phi \eta V_{z} a}{\Delta P} .
$$

The simulated results show that the permeability increases rapidly with the porosity for all types of lattices [see Fig. 3(b)]. Moreover, the Octet lattice shows significantly lower permeability when compared to the other lattices, due to the larger number of trusses that perturb the flow through the unit cell. The last parameter necessary to calculate Biot's waves in microlattices is the tortuosity $\alpha_{\infty}$. To estimate its value, we exploit the fact that for an infinitely rigid frame, the slow wave speed in the porous media is related to the wave speed in the fluid: $c_{p_{2}}=c_{w} / \sqrt{\alpha_{\infty}}$. This equation is only valid when viscous effects of the fluid are neglected. Therefore, we calculate the wave speed in the fluid part of the unit cell under the high-frequency approximation by applying the Floquet boundary conditions with a wave vector $k_{z}$ in the $z$-direction and continuity conditions in the other directions [see inset Fig. 3(c)]. The tortuosity of the three different lattices shows a similar porosity scaling with values of approximately 1.5 at $50 \%$ porosity. Assuming plane wave solutions, the dispersion relation for compressional Biot waves can be derived in terms of the complex valued inverse wave velocity $\zeta_{p}=k_{p}^{2} / \omega^{2}$ (for derivation see Appendix A),

$$
d_{2} \zeta_{p}^{2}+d_{1} \zeta_{p}+d_{0}=0
$$

where the parameters $d_{2}, d_{1}$, and $d_{0}$ are expressed in terms of generalized elastic constants and density terms

$$
\begin{aligned}
& d_{2}=P R-Q^{2}, \\
& d_{1}=-\left(P \hat{\rho}_{22}+R \hat{\rho}_{11}-2 Q \hat{\rho}_{12}\right), \\
& d_{0}=\hat{\rho}_{11} \hat{\rho}_{22}-\hat{\rho}_{12}^{2} .
\end{aligned}
$$

The dispersion relation has two complex solutions. We are interested in fast Biot waves, which are described by

$$
\zeta_{p_{1}}=\frac{-d_{1}+\sqrt{1-4 d_{0} d_{2} / d_{1}^{2}}}{2 d_{2}} .
$$

Using the values of the frame stiffness, the static permeability, and the tortuosity, we can calculate the Biot wave speeds for the analyzed microstructures (see Fig. 2). The predictions of Biot's theory based on the derived parameters agree well with the experimental data [Figs. 2(a)-2(c)] but slightly overestimate the reached speeds. We attribute this to the fact that one main assumption of Biot's theory is isotropy, which is not valid for the analysed cubic lattice materials given their orthotropic elastic response. From the imaginary part of the derived complex wave numbers, $\operatorname{Im}\left(k_{p 1}\right)$, we estimate the 
wave attenuation $\eta_{p 1}=\operatorname{Im}\left(k_{p 1}\right)$. The calculated attenuation values reproduce well the slight increase in attenuation over the considered frequency range, as found in the experimental measurement [see Figs. 2(d) and 2(f)]. However, they underestimate the attenuation especially for low porosities. This is certainly related to the fact that we use a lossless material model for the solid. For a better fit to the experimental values, viscous effects in the solid need to be included.

\section{Direct finite element calculations}

We also perform direct finite element simulations of water-saturated microlattices, including anisotropy. We model both the solid and the fluid parts of the lattices in Comsol Multiphysics coupling the two phases by pressure conditions at their interfaces. We perform a mesh convergence study in Comsol to ensure accurate results independent from the adopted discretization. We neglect any viscous coupling of the frame to the fluid. This simplification is justified by the fact that, in the investigated frequency region, the inertial effects dominate. We use cubic unit cells for each lattice and apply Floquet-type boundary conditions for the solid and fluid parts. We solve for the first eigenfrequencies of the coupled system for a given wave vector $\boldsymbol{k}=\left[\begin{array}{lll}0 & 0 & k_{z}\end{array}\right]$. At small wave numbers, the first four modes correspond to two degenerate shear modes, the slow Biot and the fast Biot waves, respectively. The simulated fast wave speeds match well with the experimental data with a significant improvement with respect to the analytical prediction [Figs. 2(a)-2(c)]. The small differences between the

(a)
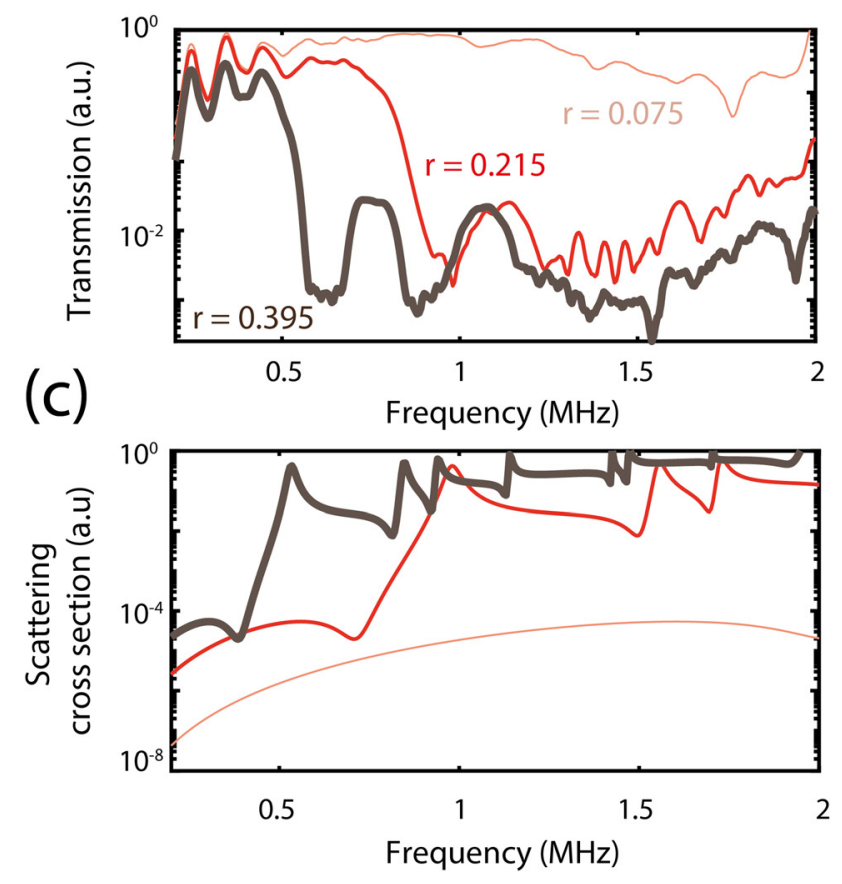

finite element simulations and the theoretical predictions by Biot's theory are due to their different assumptions. These include the assumption of material isotropy in Biot's theory. Another source of discrepancy is the viscous dissipation of the fluid, which is accounted for in Biot's theory, but not in the finite element simulation.

\section{HIGH FREQUENCY PROPAGATION}

\section{A. Experimental investigation of high frequency propagation}

To investigate the limitations of the Biot's description, we study the wave propagation at intermediate frequencies (up to $2 \mathrm{MHz}$ ) in our microlattice materials (Fig. 4). We send longer (ten cycles) chirps between $0.2 \mathrm{MHz}$ and $2 \mathrm{MHz}$ through the sample. The large hydrophone surface measures only the coherent part of the propagating wave, as the scattered wave shows an incoherent phase in the hydrophone plane, which is cancelled during integration. ${ }^{29}$ Moreover, we average over repeated measurements at slightly different positions to decrease the influence of intensity fluctuations. We show examples of the transmission curves, for Diamond lattices with truss radius $r=0.075 \mathrm{~mm}, 0.215 \mathrm{~mm}$, and $0.395 \mathrm{~mm}$ and $L=1 \mathrm{~mm}$, corresponding to porosities of $97.8 \%, 84.5 \%$, and $56.1 \%$ [Fig. 4(a)]. The initial oscillations of the transmission function are due to the finite size of the measurement line between transducer and hydrophone. The transmission spectrum of the two lowest porosity structures is characterized by an initial flat region followed by a sharp cut-off frequency that decreases for decreasing porosity

(b)

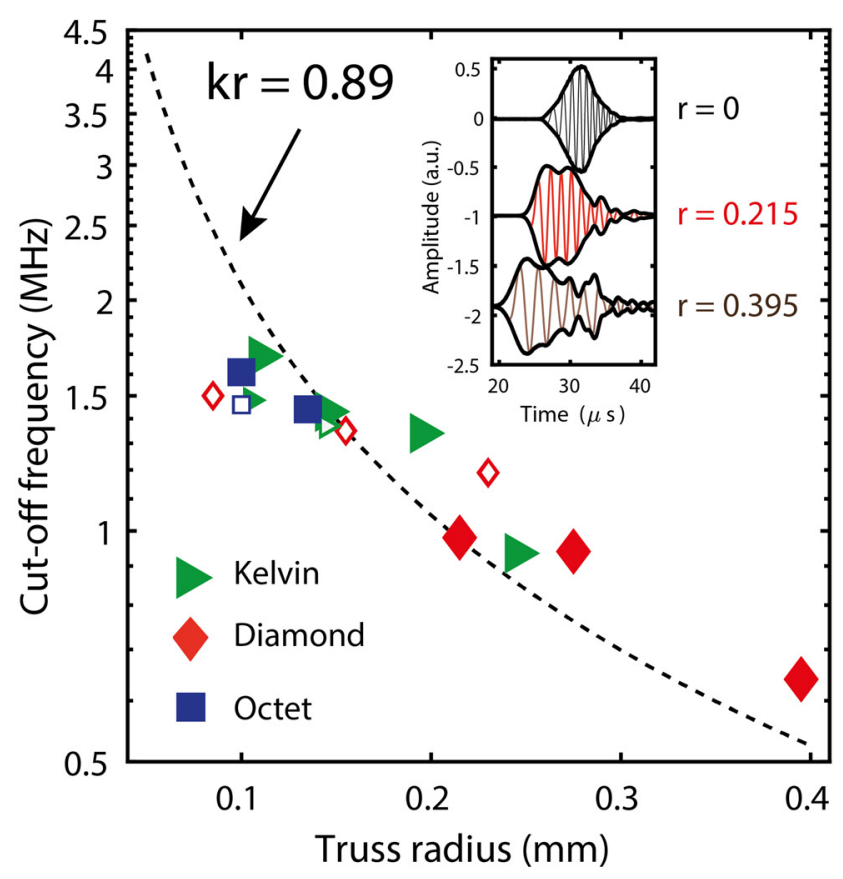

FIG. 4. (Color online) Scattering cut-off frequencies of microlattices. (a) Transmission through Diamond lattices of $r=0.075,0.215$, and 0.395 mm (thicker lines for larger radius). (b) Experimental cut-off frequencies for different lattices (markers) compared to the theoretical predicted cut-off at $k r=0.89$. Hollow markers correspond to $L=0.66 \mathrm{~mm}$ and solid markers to $L=1 \mathrm{~mm}$. The inset shows the normalized transmitted chirp signals for the three structures in (a). (c) Calculated scattering cross-section for the radii $r=0.075,0.215$, and $0.395 \mathrm{~mm}$ (thicker lines for larger radius). 
from $0.95 \mathrm{MHz}$ to $0.6 \mathrm{MHz}$. We define the cut-off frequency as the frequency at which transmission drops by $6 \mathrm{~dB}$ with respect to the low frequency transmission at $200 \mathrm{kHz}$. The biggest drop in transmission of the coherent wave is approximately two orders of magnitude for the tested lattice samples. The high porosity sample does not show any cut-off in the analyzed frequency region. We measure all lattice types and find the cut-off frequency between $0.6 \mathrm{MHz}$ and $1.7 \mathrm{MHz}$ [Fig. 4(b)].

\section{B. Scattering model}

To gain insights into the origin of the cut-off frequency, we employ a simplified scattering model of an elastic cylinder of radius $r$ and length $L$ immersed in a loss-less fluid ${ }^{32}$ (see details in Appendix B). This approach captures well the geometry of the truss; however, it does not include any stress carried by the frame as all scatterers are independent. We analyze the scattering cross section $\sigma_{s}$, which is indicative for the amount of energy transported via scattered waves,

$$
\sigma_{s}=\left|\frac{-L}{\pi} \frac{\sin (\Delta)}{\Delta} \sum_{m=0}^{\infty} \epsilon_{m} \sin \left(\eta_{m}\right) \times e^{-i \eta_{m}} \times \cos (m \theta)\right|^{2} .
$$

Here, the scattering direction angle $\theta=0$ results in the forward scattering cross-section, $\sigma_{s f}$, and $\theta=\pi$ in the back-scattered cross-section, $\sigma_{s b}$. In the case of a cylinder, $\Delta$ $=k L \cos \pi / 2$ with the wave number $k_{0}=\omega / c_{w}$. The derivation of the scattering phase angle, $\eta_{m}$, is detailed in the Appendix B. In Fig. 4(c), we show the forward scattering cross-section for the truss configurations in Fig. 4(a). The first peak of the cross-section agrees well with the experimentally measured cut-off frequency of the lattice for the larger radius samples. In general, the first peak is associated with the first acoustic Mie-resonance. ${ }^{32}$ Above the frequencies of this first peak, the scattering cross-section stays on a high level, which explains the permanent drop in signal transmission that does not recover past the cut-off frequency [see Fig. 4(a)]. Interestingly, the simple scattering model can very well be used to describe the cut-off for the various considered lattices [Fig. 4(b)] even though it neglects multiple scattering, the influence of the frame, and the orientation of the truss elements. The first acoustic Mie-resonance in this system can be described by a line of constant $k r \approx 0.89$, which matches all lattice systems despite their varying topologies and porosities (Fig. 4). In particular, the model is able to describe the cut-off frequencies of lattices independent of the truss length $L$ and hence unit cell size $a$. This is a strong indication that the observed phenomena is related to Miescattering governed by the truss-radius rather than Braggscattering, which is instead related to the periodicity of the unit cell.

\section{Group velocities}

In addition to the drastic dip in transmission by the strong scattering, we observe that the arriving ultrasonic pulses are highly dispersed (see inset of Fig. 4). We experimentally investigate the lattice dispersive properties by measuring the pulse group velocity. We filter the transmitted signals to a narrow frequency region $(0.1 \mathrm{MHz})$ using a second order Butterworth filter in MATLAB and fit an envelope function to the resulting Gaussian pulse. The group velocity, $v_{g}$, is then calculated for every frequency step as a function of the time difference, $\Delta t$, between the arrival of the maximum amplitude peak in the porous medium of length $l$ and the maximum amplitude peak through pure water

$$
v_{g}=\frac{c_{w} l}{\Delta t c_{w}+l} .
$$

The experimentally measured group velocity $v_{g}$ shows a pronounced drop for the higher density lattices (Fig. 5). The lowest measured speeds of the low and medium porosity lattices are approximately $1100 \mathrm{~m} / \mathrm{s}$ and therefore much lower than the speed of sound in the solid or in the fluid constituents. To calculate the group velocity based on scattering theory, we employ a multiple scattering model ${ }^{20}$ that describes the effective wave number $k$ as a function of the forward and backward scattering amplitudes $\left(f_{s f}\right.$ and $f_{s b}$; for details, see Appendix B)

$$
k=k_{0}^{2}+4 \pi n_{0} f_{s f}+\frac{4 \pi^{2} n_{0}^{2}}{k_{0}^{2}}\left(f_{s f}^{2}-f_{s b}^{2}\right)
$$

where $n_{0}=1-\phi / \pi r^{2} L$ is the number of cylinders per unit volume and $k_{0}=\omega / c_{w}$ is the wave number in pure water. From the effective wave number $k$, we calculate the frequency dependent group velocity by $v_{g}=d \omega / d k_{z}$ in the $z$ direction. The theoretical model shows good agreement with the high and medium porosity sample in predicting the frequency region of the lowest group velocity, even though it overestimates the decrease in velocity (see Fig. 5). This discrepancy can be explained noting that the experimental measurements of low group velocity pulses is difficult,

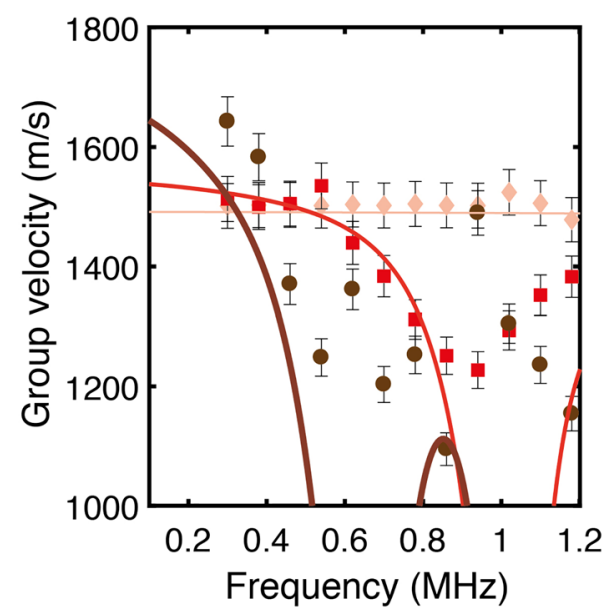

FIG. 5. (Color online) Group velocity of Diamond lattices with varying densities. We show theoretically predicted group velocities based on multiple scattering theory (solid lines) and experimental measurements (markers) for the Diamond lattices with $r=0.395 \mathrm{~mm}$ (brown, circles), $r=0.215 \mathrm{~mm}$ (red, squares), and $r=0.075 \mathrm{~mm}$ (orange, Diamond). Error bars represent the finite frequency width of the Gaussian filter that was applied to the experimental data. 
especially as they occur in regions with very low overall transmission.

\section{Discrete model}

The ability to explain the cut-off frequency by means of scattering theory suggests that the occurring phenomena do not depend strictly on periodicity of the lattice. To validate this hypothesis, we perform extended frequency fluid/solid Bloch-FE simulations of the Diamond structures (see results in Fig. 6). We focus on modes in the $z$-direction given that our experimental setup excites and measures only modes that are symmetric in the transducer plane. Therefore, we investigate the band-diagrams selecting only the modes with a large average eigenvector magnitude in the $z$ direction, both in solid and fluid displacement fields: $M_{z}=1 / 2 \int_{V_{s}}\left(\left|\hat{u}_{z}\right| /|\hat{\boldsymbol{u}}|\right) \mathrm{d} V+1 / 2 \int_{V_{f}}\left(\left|\hat{U}_{z}\right| /|\hat{\boldsymbol{U}}|\right) \mathrm{d} V$. Here, $M_{z}$ is a normalized effective displacement. During integration, the $z$-component of the displacement fields of solid and fluid over the unit cell volume effectively cancels all asymmetric modes. We analyze the phase $\mathfrak{P}$ of the occurring modes as the sign of the ratio of displacement fields, which dramatically simplifies the band-structures (see Fig. 6). The band diagrams for the three different lattices clearly show the propagation of non-dispersive slow and fast Biot waves at low frequencies (Fig. 6). The cut-off frequency $f_{c}$ agrees extremely well with the termination of the in-phase wave mode in the lower porosity lattices [Figs. 6(b) and 6(c)], which dominates the energy transport in the poroelastic material. Interestingly, the fast mode terminates above the first band folding in the intermediate porosity lattice, Fig. 6(b), and below the first folding for the low porosity lattice,
Fig. 6(c). This behaviour contradicts the behaviour of a simple phononic crystal with lattice periodicity $a$, where the gap always opens at the edge of the first Brillouin zone $k_{z}=\pi / a$. In addition, we find a narrow band-gap for the intermediate porosity lattice, which stems from the hybridization with a bending mode of the truss. ${ }^{33}$

\section{CONCLUSION}

We showed that periodic microlattices undergo a transition from a classic poroelastic effective medium description at low frequencies to a strongly scattering medium at high frequencies. Surprisingly, the frequency cut-off, which marks the limits between both descriptions, is independent of the lattice periodicity, but depends solely on the truss diameter. This result is counterintuitive when compared to classic results in multiple scattering theory ${ }^{17}$ of close packed granular media, which predicts a dependence on the lattice periodicity as described by the grain radius. The main difference in the studied microlattice materials is that their periodicity is independent of the radius, which allows for a separate treatment of periodicity and radius related effects. Biot's theory allows the description of the low frequency propagation regime, in which waves travel with small dispersion and with speeds in between pressure wave speeds in the solid and the fluid. Above the cut-off frequency, we find strong signal attenuation and exceedingly low group velocities. Such low group velocities cannot be explained by effective theories as they rely on the multiple scattering pathways through the porous material. The obtained results give insights into cross-over phenomena in acoustics that can inspire analogies to electronic or optical systems. Moreover, (a)
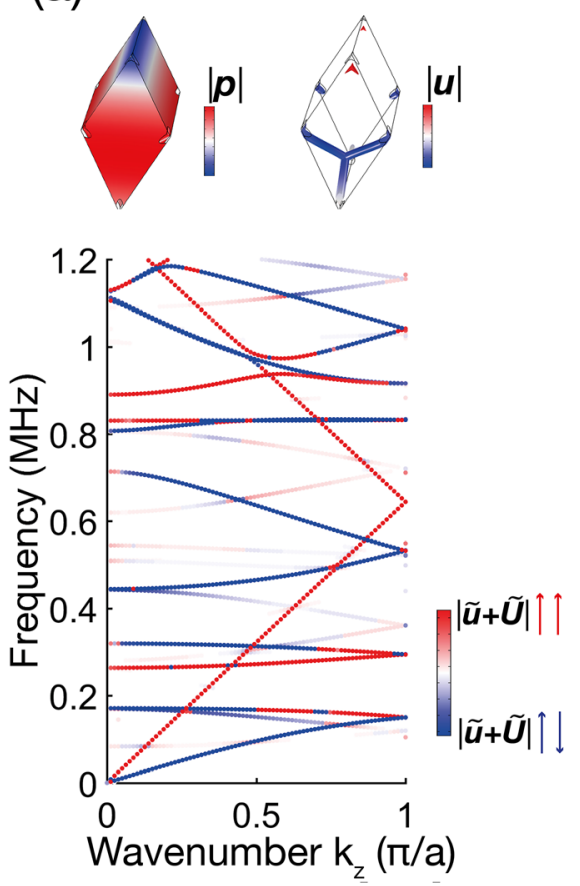

(b)

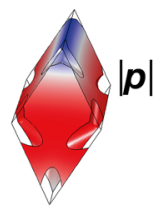

(c)

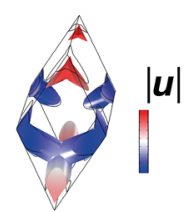

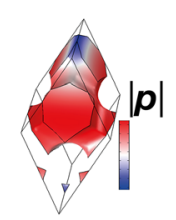

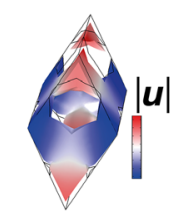

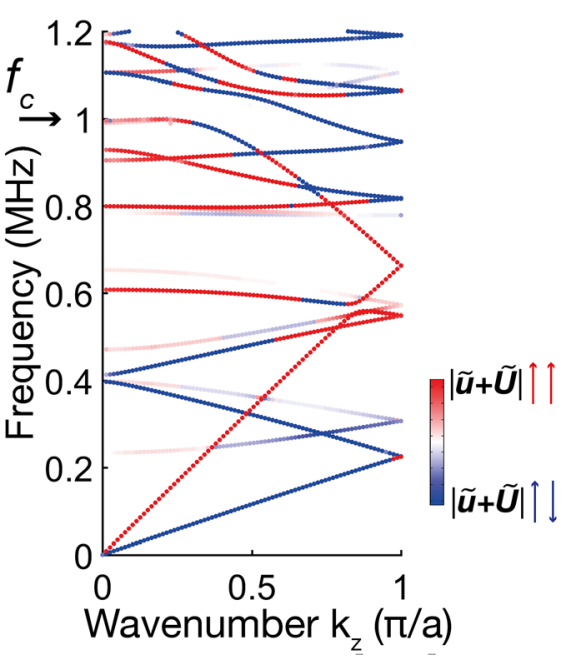

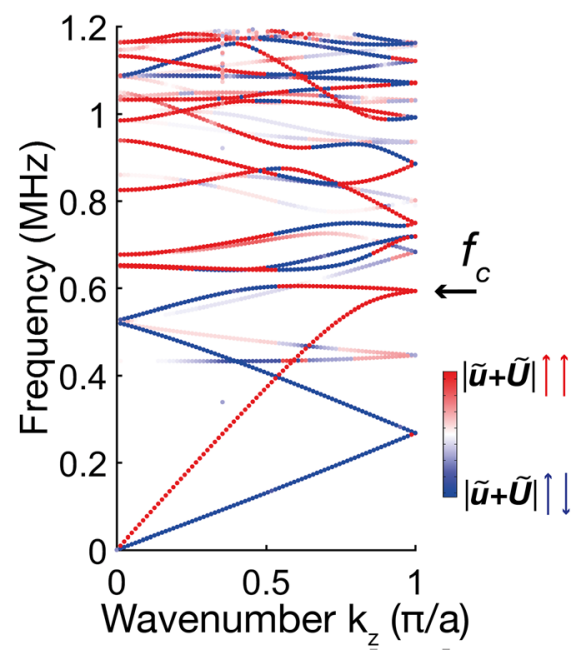

FIG. 6. (Color online) Band structures for fluid saturated Diamond lattices with (a) radius $0.075 \mathrm{~mm}$, (b) radius $0.215 \mathrm{~mm}$, and (c) radius $0.395 \mathrm{~mm}$. The color scales represent the normalized magnitude of solid and fluid displacements in the $z$-direction over the full unit cell. We show in-phase modes in red and outoff-phase modes in blue. 
the interpretation of the acoustic footprint of natural porous materials such as bones or sandstone can benefit from a precise understanding of the present acoustic regime.

\section{ACKNOWLEDGMENTS}

Funding for this research was provided by the Swiss National Science Foundation, Grant No. 164375.

\section{APPENDIX A: DERIVATION OF BIOT WAVE SPEEDS}

There exist two fundamental frequency regions: (i) a low frequency region, where the fluid can freely move between the pores, and in which the viscous forces dominate and (ii) a higher frequency region, where inertial forces are dominant, as the fluid is essentially locked to the frame movement. We restrict our investigation to the higher frequency regime, dominated by the inertial behaviour. Here we follow the derivation from Ref. 31 to obtain the equations of motion. We can start by writing the linearized equations of the solid, $\boldsymbol{e}$, and fluid, $\boldsymbol{\varepsilon}$, strain,

$$
\begin{aligned}
& \boldsymbol{e}=\frac{1}{2}\left(\nabla \hat{\boldsymbol{u}}+(\nabla \hat{\boldsymbol{u}})^{T}\right), \\
& \boldsymbol{\varepsilon}=\frac{1}{2}\left(\nabla \hat{\boldsymbol{U}}+(\nabla \hat{\boldsymbol{U}})^{T}\right),
\end{aligned}
$$

where $u_{i}$ denotes the averaged solid particle displacement and $U_{i}$ denotes the average fluid particle displacement defined by the averaging operation over the unit cell domain,

$$
\begin{aligned}
& \hat{\boldsymbol{u}}(\boldsymbol{x}, t)=\frac{1}{V^{s}} \int_{V^{s}} \boldsymbol{u}^{s}\left(\boldsymbol{x}^{\prime}, t\right) \mathrm{d} V, \\
& \hat{\boldsymbol{U}}(\boldsymbol{x}, t)=\frac{1}{V^{f}} \int_{V^{f}} \boldsymbol{U}^{f}\left(\boldsymbol{x}^{\prime}, t\right) \mathrm{d} V .
\end{aligned}
$$

The primary variable describing the mechanical properties of porous material is the porosity, which describes the ratio of pores $V^{f}$ (fluid volume) with respect to the bulk volume $V^{f}+V^{s}$ (fluid and solid volume) of the unit cell

$$
\phi=\frac{V^{f}}{V^{f}+V^{s}} .
$$

Using these definitions and applying continuity and constitutive equations and assuming harmonic motion with angular frequency $\omega$, we can write down the governing equations of motion, see Eqs. (2) and (3). In particular, we may express the elastic parameters

$$
\begin{aligned}
& A=\frac{\phi K_{l}+(1-\phi) K_{f}\left(1-\phi-\frac{K_{l}}{K_{s}}\right)}{\beta}-\frac{2}{3} G, \\
& Q=\frac{\phi K_{f}\left(1-\phi-\frac{K_{l}}{K_{s}}\right)}{\beta}, \\
& R=\frac{\phi^{2} K_{f}}{\beta},
\end{aligned}
$$

$$
P=A+2 G,
$$

where $\beta$ denotes the so-called effective porosity, defined by $\beta=\phi+K_{f} / K_{s}(1-\phi)$. The density terms are expressed as

$$
\begin{aligned}
& \rho_{12}=-\left(\alpha_{\infty}-1\right) \phi \rho_{f}, \\
& \rho_{11}=(1-\phi) \rho_{s}-\rho_{12}, \\
& \rho_{22}=\phi \rho_{f}-\rho_{12} .
\end{aligned}
$$

Here, $\alpha_{\infty}$ denotes the tortuosity $\left(\alpha_{\infty}>1\right)$, which is a purely geometrical parameter. This parameter can be interpreted as the slowdown of sound due to the added path in the porous structure. In fact, it can be shown ${ }^{34}$ that in the limit of an infinitely stiff frame, the fast compressional wave does not exist and there is only a slow compressional wave with the wave speed $c_{p 2}=c_{w} / \sqrt{\alpha_{\infty}}$. To account for the dynamic interaction between lattice and fluid movement, the density terms are corrected as a function of frequency

$$
\begin{aligned}
& \hat{\rho}_{12}=\rho_{12}-i \hat{b}(\omega) / \omega, \\
& \hat{\rho}_{11}=\rho_{12}-i \hat{b}(\omega) / \omega, \\
& \hat{\rho}_{22}=\rho_{22}-i \hat{b}(\omega) / \omega .
\end{aligned}
$$

The frequency dependent dissipation function $\hat{b}(\omega)$ $=b_{0} \sqrt{1-i f / 2 f_{r}}$ is related to the dynamic permeability $\hat{\kappa}(\omega)$ of the porous material

$$
\hat{b}(\omega)=b_{0}\left(\frac{\kappa_{0}}{\hat{\kappa}(\omega)}+i \frac{f}{f_{c}}\right) .
$$

Furthermore, we define the viscous damping parameter as

$$
b_{0}=\frac{\eta_{f} \phi^{2}}{\kappa_{0}},
$$

where $\eta_{f}$ is the fluid viscosity, and $k_{0}$ the static permeability according to a Darcian flow. The dynamic interaction is characterized by the rollover frequency or Biot frequency

$$
f_{r}=\frac{\eta_{f} \phi}{2 \pi \rho \kappa_{0} \alpha_{\infty}}
$$

which can be interpreted as a ratio between viscous and inertial forces. We may rewrite the displacement of the solid and the liquid components in terms of scalar and vector potential functions

$$
\begin{aligned}
& \hat{\boldsymbol{u}}=\nabla \hat{\Phi}_{s}+\nabla \times \hat{\Psi}_{s}, \\
& \hat{\boldsymbol{U}}=\nabla \hat{\Phi}_{f}+\nabla \times \hat{\Psi}_{f} .
\end{aligned}
$$

Using plane-wave like solutions for the potentials

$$
\begin{aligned}
& \hat{\Phi}_{s, f}=\tilde{\Phi}_{s, f} e^{i \boldsymbol{k} \cdot \boldsymbol{x}}, \\
& \hat{\Psi}_{s, f}=\tilde{\Psi}_{s, f} e^{i \boldsymbol{k} \cdot \boldsymbol{x}},
\end{aligned}
$$


and considering the propagation of longitudinal waves in one direction of the isotropic space, we can derive the dispersion relation for compressional waves in terms of the complex valued inverse wave velocity $\zeta_{p}=k_{p}^{2} / \omega^{2}$,

$$
d_{2} \zeta_{p}^{2}+d_{1} \zeta_{p}+d_{0}=0
$$

\section{APPENDIX B: ACOUSTIC SCATTERING OF FINITE ELASTIC CYLINDERS}

According to Ref. 32, the normal incidence scattering pressure field from an elastic cylinder of length $L$ and radius $r$ immersed in a fluid can be estimated as

$$
\begin{aligned}
p_{\text {scat }}= & P_{0} \frac{e^{i k r}}{r}\left(\frac{-L}{\pi}\right) \frac{\sin (\Delta)}{\Delta} \sum_{m=0}^{\infty} \epsilon_{m} \sin \left(\eta_{m}\right) \\
& \times e^{-i \eta_{m}} \times \cos (m \theta),
\end{aligned}
$$

where $\Delta=k L \cos (\pi / 2)$ and $\epsilon_{m}$ is the Neumann number. The wave numbers $\eta_{m}$ can be calculated by

$$
\begin{gathered}
\tan \eta_{m}=\left[\tan \delta_{m}\left(x_{f}\right)\right]\left[\tan \Phi_{m}+\tan \alpha_{m}\left(x_{f}\right)\right] / \\
{\left[\tan \Phi_{m}+\tan \beta_{m}\right]} \\
\tan \delta_{m}\left(x_{f}\right)=-J_{m}\left(x_{f}\right) / N_{m}\left(x_{f}\right), \\
\tan \alpha_{m}\left(x_{f}\right)=-x_{f} J_{m}^{\prime}\left(x_{f}\right) / J_{m}\left(x_{f}\right) \\
\tan \beta_{m}\left(x_{f}\right)=-x_{f} N_{m}^{\prime}\left(x_{f}\right) / N_{m}\left(x_{f}\right) \\
\tan \Phi_{m}=-\left(\rho_{f} / \rho_{s}\right) \tan \xi_{m} \\
\tan \xi_{m}=-\frac{x_{s, s}^{2}}{2}\left(\frac{A-B}{C-D}\right)
\end{gathered}
$$

where

$$
\begin{aligned}
& A=\left[\tan \alpha_{m}\left(x_{s, p}\right)\right] /\left[\tan \alpha_{m}\left(x_{s, p}\right)+1\right] \\
& B=m^{2} /\left[\tan \alpha_{m}\left(x_{s, p}\right)+m^{2}-\frac{1}{2} x_{s, s}^{2}\right] \\
& C=\left[\tan \alpha_{m}\left(x_{s, p}\right)+m^{2}-\frac{1}{2} x_{s, s}^{2}\right] /\left[\tan \alpha_{m}\left(x_{s, p}\right)+1\right] \\
& D=m^{2}\left[\tan \alpha_{m}\left(x_{s, s}\right)+1\right] /\left[\tan \alpha_{m}\left(x_{s, s}\right)+m^{2}-\frac{1}{2} x_{s, s}^{2}\right] .
\end{aligned}
$$

Here, $J_{m}(x)$ denotes a Bessel function of first kind and order $m$, and $N_{m}(x)$ a Bessel function of second kind and order $m$. The scattering phase angles $\eta_{m}, \delta_{m}, \Phi_{m} \alpha_{m}, \beta_{m}$, and $\xi_{m}$ are functions of the relative wave numbers in the solid $x_{s, s}=k_{s} r$, $x_{s, p}=k_{p} r$, and the fluid $x_{f}=k_{f} r$. The scattering pressure can be brought in a more compact form

$$
p_{\text {scat }}=P_{0}\left(e^{i k r} / r\right) f(\omega)
$$

where $f$ is denoted as the scattering amplitude of the cylinder object

$$
f(\omega)=\frac{-L}{\pi} \frac{\sin (\Delta)}{\Delta} \sum_{m=0}^{\infty} \epsilon_{m} \sin \left(\eta_{m}\right) \times e^{-i \eta_{m}} \times \cos (m \theta) .
$$

The scattering cross section is then defined as

$$
\sigma_{s}=|f(\omega)|^{2} .
$$

To calculate the frequency dependent scattering cross section of the microlattice materials, we fix the frequency dependent wave numbers, $k=2 \pi f / c_{i}$, in solid and fluid. The sum in Eq. (B13) can be terminated after 30 terms.

${ }^{1}$ M. Maldovan, "Sound and heat revolutions in phononics," Nature 503(7475), 209-217 (2013).

${ }^{2} \mathrm{M}$. Maldovan, "Phonon wave interference and thermal bandgap materials," Nat. Mater. 14(7), 667-674 (2015).

${ }^{3} \mathrm{P}$. Sheng, Introduction to Wave Scattering, Localisation, and Mesoscopic Phenomena (Springer, New York, 2006).

${ }^{4}$ J. H. Page, P. Sheng, H. P. Schriemer, I. Jones, X. Jing, and D. A. Weitz, "Group velocity in strongly scattering media," Science 271(5249), 634-637 (1996)

${ }^{5}$ T. Bückmann, N. Stenger, M. Kadic, J. Kaschke, A. Frölich, T. Kennerknecht, C. Eberl, M. Thiel, and M. Wegener, "Tailored 3D mechanical metamaterials made by dip-in direct-laser-writing optical lithography," Adv. Mater. 24(20), 2710-2714 (2012).

${ }^{6}$ M. Kadic, T. Bückmann, N. Stenger, M. Thiel, and M. Wegener, "On the practicability of pentamode mechanical metamaterials," Appl. Phys. Lett. 100(19), 191901 (2012)

${ }^{7}$ T. Bückmann, M. Thiel, M. Kadic, R. Schittny, and M. Wegener, "An elasto-mechanical unfeelability cloak made of pentamode metamaterials," Nat. Commun. 5, 4130 (2014).

${ }^{8}$ T. Bückmann, R. Schittny, M. Thiel, M. Kadic, G. W. Milton, and M. Wegener, "On three-dimensional dilational elastic metamaterials," New J. Phys. 16, 033032 (2014).

${ }^{9}$ M. A. Biot, "Theory of propagation of elastic waves in a fluid-saturated porous solid. II. Higher frequency range," J. Acoust. Soc. Am. 28(2), 179-191 (1956).

${ }^{10} \mathrm{M}$. A. Biot, "Theory of propagation of elastic waves in a fluid-saturated porous solid. I. Low-frequency range," J. Acoust. Soc. Am. 28(2), 168-178 (1956).

${ }^{11}$ Z. E. A. Fellah, J. Y. Chapelon, S. Berger, W. Lauriks, and C. Depollier, "Ultrasonic wave propagation in human cancellous bone: Application of Biot theory," J. Acoust. Soc. Am. 116(1), 61-73 (2004).

${ }^{12} \mathrm{H}$. Steeb, "Ultrasound propagation in cancellous bone," Arch. Appl. Mech. 80(5), 489-502 (2010).

${ }^{13}$ O. Kelder and D. M. J. Smeulders, "Observation of the Biot slow wave in water-saturated Nivelsteiner sandstone," Geophysics 62(6), 1794-1796 (1997).

${ }^{14}$ C. Perrot, F. Chevillotte, M. T. Hoang, G. Bonnet, F.-X. Bécot, L. Gautron, and A. Duval, "Microstructure, transport, and acoustic properties of open-cell foam samples: Experiments and three-dimensional numerical simulations," J. Appl. Phys. 111(1), 014911 (2012).

${ }^{15} \mathrm{~F}$. Chevillotte and C. Perrot, "Effect of the three-dimensional microstructure on the sound absorption of foams: A parametric study," J. Acoust. Soc. Am. 142(2), 1130-1140 (2017).

${ }^{16}$ J. F. Allard and Y. Champoux, "New empirical equations for sound propagation in rigid frame fibrous materials," J. Acoust. Soc. Am. 91(6), 3346-3353 (1992).

${ }^{17}$ L. Schwartz and T. Plona, "Ultrasonic propagation in close-packed disordered suspensions," J. Appl. Phys. 55(11), 3971-3977 (1984).

${ }^{18}$ H. Hu, A. Strybulevych, J. H. Page, S. E. Skipetrov, and B. A. van Tiggelen, "Localization of ultrasound in a three-dimensional elastic network," Nat. Phys. 4(12), 945-948 (2008).

${ }^{19}$ S. Yang, J. Page, Z. Liu, M. Cowan, C. Chan, and P. Sheng, "Ultrasound tunneling through 3D phononic crystals," Phys. Rev. Lett. 88(10), 104301 (2002).

${ }^{20}$ T. Brunet, A. Merlin, B. Mascaro, K. Zimny, J. Leng, O. Poncelet, C. Aristegui, and O. Mondain-Monval, "Soft 3D acoustic metamaterial with negative index," Nat. Mater. 14, 384-388 (2014). 
${ }^{21}$ T. Brunet, J. Leng, and O. Mondain-Monval, "Soft acoustic metamaterials," Science 342(6156), 323-324 (2013).

${ }^{22}$ V. Leroy, A. Strybulevych, M. Lanoy, F. Lemoult, A. Tourin, and J. H. Page, "Superabsorption of acoustic waves with bubble metascreens," Phys. Rev. B 91(2), 020301 (2015).

${ }^{23} \mathrm{~K}$. A. Wear, "Frequency dependence of ultrasonic backscatter from human trabecular bone: Theory and experiment," J. Acoust. Soc. Am. 106(6), 3659-3664 (1999).

${ }^{24}$ F. Luppé, J.-M. Conoir, and H. Franklin, "Scattering by a fluid cylinder in a porous medium: Application to trabecular bone," J. Acoust. Soc. Am. 111(6), 2573-2582 (2002).

${ }^{25}$ J. G. Berryman, "Single-scattering approximations for coefficients in Biot's equations of poroelasticity," J. Acoust. Soc. Am. 91(2), 551-571 (1992).

${ }^{26}$ T. Haire and C. Langton, "Biot theory: A review of its application to ultrasound propagation through cancellous bone," Bone 24(4), 291-295 (1999).

${ }^{27} \mathrm{O}$. Dazel and V. Tournat, "Nonlinear Biot waves in porous media with application to unconsolidated granular media," J. Acoust. Soc. Am. 127(2), 692-702 (2010).
${ }^{28}$ J. Legland, V. Tournat, O. Dazel, A. Novak, and V. Gusev, "Linear and nonlinear Biot waves in a noncohesive granular medium slab: Transfer function, self-action, second harmonic generation," J. Acoust. Soc. Am. 131(6), 4292-4303 (2012).

${ }^{29}$ M. L. Cowan, K. Beaty, J. H. Page, Z. Liu, and P. Sheng, "Group velocity of acoustic waves in strongly scattering media: Dependence on the volume fraction of scatterers," Phys. Rev. E 58(5), 6626-6636 (1998).

${ }^{30}$ M. F. Ashby, "The properties of foams and lattices," Philos. Trans. Ser. A. Math. Phys. Eng. Sci. 364(1838), 15-30 (2006).

${ }^{31}$ O. Kelder, Frequency-Dependent Wave Propagation in Water-Saturated Porous Media (Technische Universiteit Delft, Delft, the Netherlands, 1997).

${ }^{32}$ T. K. Stanton, "Sound scattering by cylinders of finite length. II. Elastic cylinders," J. Acoust. Soc. Am. 83(1), 64-67 (1988).

${ }^{33}$ S. Krödel and C. Daraio, "Microlattice metamaterials for tailoring ultrasonic transmission with elastoacoustic hybridization," Phys. Rev. Appl. 6(6), 064005 (2016)

${ }^{34}$ D. L. Johnson, T. J. Plona, and H. Kojima, "Probing porous media with first and second sound. II. Acoustic properties of water-saturated porous media," J. Appl. Phys. 76(1), 115-125 (1994). 